\title{
ISO 26000 : la nouvelle lingua franca de la responsabilité sociétale?
}

\author{
Jeanne Simard, Marc-André Morency, Marilyne Simard et Manon Paré \\ Laboratoire de recherche et d'intervention en gouvernance des organisations \\ Université du Québec à Chicoutimi
}

\section{INTRODUCTION}

\begin{abstract}
Depuis quelques décennies, avec l'accroissement des échanges à l'échelon planétaire, de nombreux acteurs sociaux, de nombreuses nations, ont pris conscience de l'urgence d'adopter des comportements responsables visant à promouvoir la qualité de la vie sociale et la préservation de l'environnement.
\end{abstract}

Depuis quelques décennies, avec l'accroissement des échanges à l'échelon planétaire, de nombreux acteurs sociaux, de nombreuses nations, ont pris conscience de l'urgence d'adopter des comportements responsables visant à promouvoir la qualité de la vie sociale et la préservation de l'environnement. En 1946, la Déclaration de Philadelphie, instituant le Bureau international du travail (BIT) en exposait déjà plusieurs principes directeurs ${ }^{1}$. Dans un contexte de gouvernance globale à nouveau plus réceptif à une telle réflexion, on mesurait mieux la nécessité d'encadrer les activités et le pouvoir grandissant des multinationales. On voyait avec plus d'acuité diverses problématiques sociales et écologiques résultant d'activités économiques trop faiblement régulées, ainsi que les carences de nombreux États, l'« État de droit» n'existant pratiquement pas sur de vastes portions de la planète. Face à ces nouveaux défis confrontant les communautés dans le monde, le concept de « responsabilité sociétale » $(\mathrm{RS})$, impliquant une large participation, un rôle dirigeant de la collectivité et de ses institutions publiques, assumerait un rôle mieux déterminé, en guise de contrepoids à la liberté d'entreprendre.

Mais sur quelle base, et selon quelles dimensions, la responsabilité sociétale pourrait- elle être structurée? Et de quelle façon pouvaiton élaborer une telle norme? Déjà plusieurs initiatives internationales avaient tenté de donner un cadre au concept de «responsabilité sociale », mais aucune n'était parvenue à vraiment faire consensus. On restait sur sa faim devant le banquet offert par le «self-service» normatif des entreprises jusque-là privilégié par les tenants du libéralisme économique ${ }^{2}$. Au tournant du nouveau millénaire, devant les résultats encourageants obtenus avec la Norme 14001 sur le management environnemental, l'Organisation internationale de normalisation (ISO) vit l'opportunité de réaliser une norme internationale sur la responsabilité sociétale (RS), d'aller au-delà des notions polysémiques de responsabilité sociale des entreprises (ou RSE), ou des notions générales de responsabilité que l'on trouve en morale, en éthique et en droit. ISO, en raison de son expertise dans la conduite de processus consultatifs internationaux, s'est sentie prête à tenter l'aventure. À l'issue d'un processus complexe de réflexion scientifique, éthique et internationale, sans précédent, ISO réussit à publier, en 2010, la Norme ISO 26000 sur la responsabilité sociétale visant les principes directeurs de la vie collective et la gouvernance des organisations, qu'elles soient privées ou publiques.

\footnotetext{
À l'issue d'un processus complexe de réflexion scientifique, éthique et internationale, sans précédent, ISO réussit à publier, en 2010, la Norme ISO 26000 sur la responsabilité sociétale visant les principes directeurs de la vie collective et la gouvernance des organisations, qu'elles soient privées ou publiques.
} 
Après un bref rappel du rôle de cette organisation internationale de normalisation, nous présenterons le processus de consultation ayant permis l'élaboration et l'adoption de la Norme ISO 26000. Suivra une rapide description de la norme. Il sera ensuite possible d'indiquer ce que pourra être le rôle de cette norme, comment elle favorisera l'implantation d'un développement plus responsable, respectueux de l'environnement et des communautés.
Bien qu'il soit encore un peu tôt pour en apprécier tout l'impact, la norme ISO 26000 représente actuellement un progrès significatif de la communauté internationale dans la conception d'un développement valable sur le plan éthique. Dans cette mesure, les organisations, les gestionnaires, aussi bien dans le public que dans le privé, ont tout intérêt à en réaliser les potentialités et à anticiper ses impacts.

\section{LE PROBLÈME MONDIAL : CONFUSION CONCEPTUELLE ET ABSENCE D'AUTORITÉ RÉGULATRICE}

\section{Lorsque des gestionnaires, des politiciens, des fonctionnaires, des universitaires, définissent ce qu'ils entendent par \\ « responsabilité sociale ou sociétale », on peut s'attendre à presque autant de définitions ou de visions différentes qu'il y a d'interlocuteurs.}

Lorsque des gestionnaires, des politiciens, des fonctionnaires, des universitaires, définissent ce qu'ils entendent par "responsabilité sociale ou sociétale », on peut s'attendre à presque autant de définitions ou de visions différentes qu'il y a d'interlocuteurs. C'est un peu ce qu'exprime le dicton populaire «À chacun sa vérité ». Et cette problématique s'accroit encore lorsque l'on change de pays, de culture ou d'environnement ${ }^{3}$. Ces différences se comprennent très bien si l'on considère que la notion de responsabilité sociale des entreprises (RSE) n'est pas à l'origine une notion scientifique, c'est-à-dire qu'elle n'a pas une fonction analytique ayant pour dessein de comprendre le monde, la réalité observée. La notion de responsabilité sociale des entreprises est souvent dépeinte «comme un moyen de doter la globalisation de règles sociales et écologiques, tout en laissant les opérateurs économiques choisir à cette fin celles qui leur sont le mieux adaptées $»$. Mais elle devient de ce fait une notion polysémique qui se trouve «construite» différemment par de nombreux acteurs en conflit, en fonction d'une variété de conceptions du monde social, des limites de la responsabilité sociale des entreprises dans la détermination du bien commun (conditions de travail, protection de l'environnement, développement scientifique et technologique, etc. $)^{5}$. Philippe Chalmin en rajoute en rappelant que dans la sphère marchande mondialisée aucune autorité n'est encore en mesure d'instaurer une quelconque forme de normativité, de vision commune, et encore moins de régulation ${ }^{6}$.

Sous-jacente aux diverses variantes, on trouve une conception dogmatique de la responsabilité sociale des entreprises dominantes, celle du néolibéralisme économique, de l'ordre spontané et infaillible du Marché. Obéissant au mécanisme comptable de la Fair value, l'entreprise devrait, dans un contexte de globalisation, se consacrer à accroitre l'avoir des actionnaires. Sa « responsabilité » (si l'on peut encore employer ce terme) se limite à ce seul objet, même si des actions de philanthropie ou de mécénat peuvent à l'occasion être envisagées ${ }^{7}$. D'ailleurs on entend très souvent dans les médias, ou lors d'entrevues avec des personnalités du monde des affaires, des discours qui en disent long sur cette représentation; «l'entreprise est la propriété des actionnaires » ou « les membres du conseil d'administration sont les fiduciaires des actionnaires $»^{8}$. De l'autre côté du spectre, on peut considérer l'entreprise comme une personne morale, ce qui est possible en droit, mais à la limite, comme un citoyen à part entière, ce qui est excessif. Comme personne morale, elle a la capacité de prendre des engagements moraux vis-à-vis les impacts environnementaux et sociaux de ses activités, et ce faisant, de considérer diverses parties prenantes. Pour réussir dans ce large créneau d'opportunités et de contraintes, 
elle doit aller au-delà des simples obligations nationales d'origine légale et règlementaire pour établir un dialogue avec ses parties prenantes : actionnaires, salariés, fournisseurs, ONG, gouvernements, communautés, etc. Entre ces deux pôles, relativement aux attentes à l'endroit des organisations, on trouve toute une série de situations intermédiaires, de compromis, en fonction des contextes d'opération qu'offre la réalité mondiale.

Mais peu importe la définition de la responsabilité sociale des entreprises qui sera retenue par les différents intervenants, elle ne saurait jamais offrir une vision globale et en profondeur de la réalité du phénomène, c'est-àdire, des enjeux sociaux en cause et des idéologies sous-jacentes ${ }^{9}$. La notion de responsabilité sociale des entreprises sera forcément modulée en fonction des notions polysémiques d'expansion, de croissance et de développement qui ont cours. Il s'agit d'une autre source de ces visions extrêmement contrastées où la confusion règne en maître.

Quelques initiatives internationales ont tenté, sans grand succès, de procurer un cadre au concept de responsabilité sociale des entreprises. On pense ici aux efforts de l'Organisation des Nations Unies, de l'Organisation internationale $\mathrm{du}$ travail (OIT) et de l'Organisation de coopération et de développement économique (OCDE). Faute de consensus, cette notion «optimiste» n'a abouti qu'à la frustration généralisée des parties prenantes, à l'immobilisation des cadres publics, c'est-à-dire à leur défaut d'encadrer. En fait, le monde se cherche toujours un langage commun, ce qui va bien audelà de l'usage généralisé d'une langue particulière. Un langage existe lorsque ses référents conceptuels sont du même ordre, lorsque les définitions de situation reposent sur des concepts et des cadres appropriés, permettant de créer une même réalité. En somme, un langage existe lorsque les acteurs possèdent une culture commune. Or, ce qui existe actuellement, c'est une ébauche d'intérêts vitaux communs, certains mécanismes économiques et techniques communs, mais en aucune façon un ordre institutionnel partagé, un ensemble d'objectifs poli- tiques, économiques, environnementaux commun, bref un État de droit planétaire.

Ce constat de la confusion des termes et des effets de réalité du langage nous amènent à revisiter un théorème célèbre en sociologie. Un théorème pose un principe auquel n'échappe aucun élément de la réalité en cause. William I. Thomas a bel et bien formulé un véritable théorème sociologique en affirmant que «si les humains définissent une situation comme réelle, en certains termes, cela a, et aura, des effets réels $»^{10}$. Un système de pensée, comme toute prophétie, possède un pouvoir autoréalisateur. Il oriente les comportements de telle sorte qu'ils tendent à faire advenir ce qu'il annonce, que ce soit par un effet de l'autorité de celui qui énonce la prophétie ou par la focalisation autonome des esprits sur le «réel» ainsi affirmé ${ }^{11}$. Même la pensée magique n'est pas dénuée de sens, car elle produit du sens, et ce sens produit des effets réels. Plusieurs modèles économiques ont ce caractère de la pensée magique; l'imagerie associée à la «main invisible du marché » le montre à l'évidence ${ }^{12}$.

Les normes constituent un corpus prophétique de cette nature, cela va de soi. Lorsqu'une norme est adoptée, avant même d'être renforcée, elle a des effets réels du seul fait de sa diffusion. La force agissante du discours normatif se mesure à sa capacité de faire croire à la réalité de ce qu'il décrit, à la vérité de ce qu'il énonce. C'est tout l'intérêt de la norme ISO 26000 : ce n'est pas qu'une norme, c'est un langage, et là où il sera adopté, il aura des effets réels, comme une nouvelle lingua franca $^{13}$. Elle contribuera à faire surgir une réalité commune là où il n'y avait que confusion et neutralisation mutuelle de concepts opposés.

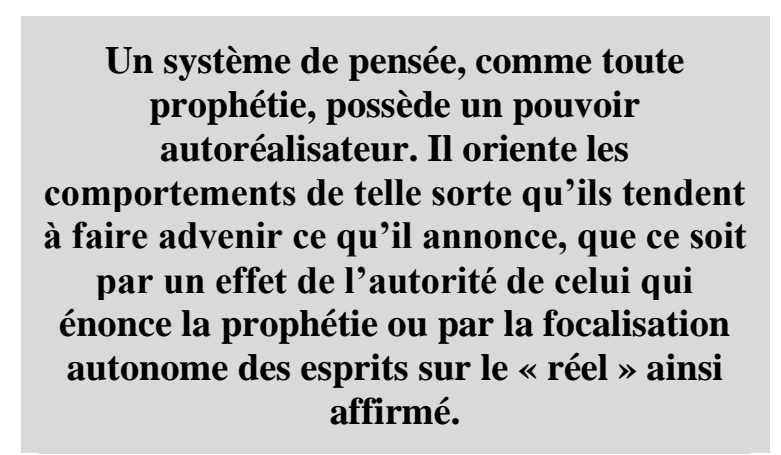




\section{L'ORGANISATION INTERNATIONALE DE NORMALISATION (ISO)}

ISO est un organisme international, mais non gouvernemental, de standardisation industrielle et commerciale, créé en 1947 pour appuyer l'effort de reconstruction de 1'après-guerre. Ayant son siège social à Genève, il est devenu, au fil des ans, le plus grand producteur et éditeur de normes privées au monde. ISO déploie plus de 200 comités techniques qui ont, face aux besoins du marché, élaboré des milliers de normes internationales portant sur des aspects divers de la pratique des entreprises, dans les secteurs de l'agriculture, de la construction, du transport, des dispositifs médicaux, des technologies de l'information et de la communication, de la mécanique, de la fabrication et de la distribution.

$\mathrm{Si}$, à l'origine, ISO publiait surtout des normes techniques pour la certification de produits, de procédés de fabrication, voire de services, son champ d'intérêt s'est élargi pour en arriver à couvrir les processus et systèmes de gestion; par exemple, la Norme 9001 sur le management de la qualité et ses dérivés, la Norme 14001 sur le management de l'environnement. En formulant, en 2011, la Norme 26000 sur la responsabilité sociétale, ISO est allée encore plus loin.

Sur le plan pratique, l'Organisation internationale de normalisation élabore les normes sous la forme d'un « document établi par consensus » en impliquant l'ensemble des parties intéressées ou divers partenaires. Le Canada, entre autres, est représenté par le Conseil national de normalisation $(\mathrm{CNN})$. Chaque pays a une voix dans les procédures de vote. Selon Benyekhlef :

L'organisme de normalisation ne peut créer de spécifications techniques ex nihilo. La norme doit s'intégrer dans la réalité. Le normalisateur doit donc porter une attention particulière au réel et observer patiemment ce qui existe. La norme technique doit, aussi, être accessible au public, être disponible matériellement et faire l'objet d'une publication ${ }^{14}$.

Un mécanisme de certification complète la normalisation lorsqu'il s'agit de champs techniques. Il permet d'attester, après une vérification indépendante, qu'une production, qu'un service, qu'un système, satisfait aux exigences d'une norme ISO. Par contre, il est important de rappeler que les normes formulées par ISO n'ont force de loi ou de règlement ni dans les pays ni dans les organisations. Leur mise en application est en principe volontaire.

\section{PROCESSUS D'ÉLABORATION DE LA NORME ISO 26000}

Devant l'intérêt sans cesse croissant en matière de responsabilité sociétale et la confusion des définitions, le besoin de créer une norme internationale dans ce domaine se fit pressant. En 2001, COPOLCO, le Comité ISO pour la politique en matière de consommation, fut chargé d'examiner la faisabilité d'une norme sur la responsabilité sociétale. En 2003, à la suite des recommandations du COPOLCO, le Bureau de gestion technique de l'ISO créait un comité composé de multiples parties prenantes, afin de réaliser une analyse complète des initiatives et enjeux en matière de responsabilité sociétale dans le monde.

En 2004, ce comité ayant fait part de ses recommandations, ISO crée un groupe de travail sur la responsabilité sociétale, ISO/GT RS, et le charge d'élaborer la future norme ISO 26000. Même pour une organisation comme ISO, habituée à divers processus de rédaction international et multipartite, l'élaboration de la norme sur la responsabilité sociétale représente un défi de taille. Un tel projet, d'une ampleur sans précédent, oblige ISO à modifier son processus rédactionnel habituel, d'y incorporer des représentants de la société civile, non convoqués habituellement ${ }^{15}$. Le processus devait en quelque sorte résoudre le problème de la confusion des termes tels qu'ils existent au départ, voire durant le processus consultatif en cours, dans la conscience des participants; ces termes étant ceux-là mêmes qui seront déterminés plus adéquatement dans une définition commune. 
Chaque pays membre d'ISO pouvait désigner jusqu'à six représentants, appartenant à six catégories de parties prenantes: l'industrie, le gouvernement, les organisations non gouvernementales (ONG), les travailleurs, les consommateurs et les «autres» (services, conseil, recherches, etc.). Les organismes internationaux tels que les Nations Unies, l'OIT, le GRI, l'OCDE, pouvaient également déléguer des experts afin d'y être représentés. La direction du groupe de travail fut confiée à deux organismes nationaux de normalisation, ceux de Suède et du Brésil. Au moment de sa publication, quelque 99 pays, 450 experts et plus de 40 organisations internationales avaient participé à l'élaboration de la norme, au terme d'un effort international remarquable. Il aura fallu cinq ans de travaux et huit réunions internationales, dont une à Québec en mai 2009, pour élaborer et rédiger cette norme. Il est facile d'imaginer les défis posés par la participation au processus de consultation d'un pareil nombre de parties prenantes. Quelque 25000 observations écrites ayant été reçues, il fallait concilier des positions parfois radicalement opposées. Comme pour les autres normes ISO, un consensus de $75 \%$ des membres votants permettait de valider l'adoption de la norme. De fait, seulement cinq représentants ont

\section{STRUCTURE ET CONTENU DE LA NORME}

Comme la norme ISO 26000 fait un peu plus de 125 pages, il s'agit avant tout, dans cet article, d'en présenter la structure et les principales lignes directrices.

Dès le départ, mentionnons que les rédacteurs de cette norme se sont démarqués par rapport à l'utopie néolibérale et à certains de ses dogmes (infaillibilité du marché sans limites régulatoires, bienfaits généralisés de la concurrence universelle, privatisation des services publics, dérèglementation du travail, libre circulation des capitaux et des marchandises ${ }^{17}$ ). Les rédacteurs réfèrent plutôt aux concepts de justice sociale, de solidarité, s'inspirant de textes comme la Déclaration de Philadelphie de $1944^{18}$, soit la première déclaration internationale des droits à vocation universelle; également de la Déclaration de l'OIT sur la justice sociale : pour une mondialisation équitable de 2008. Le social voté contre l'adoption d'ISO 26000 : ceux des États-Unis, Cuba, Inde, Turquie et Luxembourg. Le lancement officiel de la norme ISO 26000 : 2010, Lignes directrices relatives à la responsabilité sociétale eut lieu le $1^{\mathrm{er}}$ novembre 2010. Corinne Gendron a bien fait ressortir le caractère innovateur du processus et de son résultat :

Il est clair en effet que ce qui peut sembler une évolution naturelle — de la qualité, à l'environnement, à la responsabilité sociale - constitue en fait une révolution presque contre nature alors qu'ISO fait irruption dans le champ normatif et substantif en laissant de côté les procédures et les systèmes de gestion qui ont été sa marque et sur lesquels elle a bâti sa crédibilité. Avec la norme 26000 , ISO ne dit plus seulement comment faire, mais bien quoi faire ${ }^{16}$.

Cette norme, contrairement aux autres normes ISO, n'est pas destinée à la certification. Elle n'énonce pas des méthodes pouvant devenir des outils de gestion. Elle énonce plutôt une vision, des valeurs, des principes, des lignes directrices qui orientent les gestionnaires et leurs vis-à-vis dans leurs interactions.

n'est plus un simple moyen d'atteindre les objectifs de rendement des organisations, mais apparaît plutôt comme la finalité de leur existence, de leur raison d'être. L'État de droit et ses institutions démocratiques retrouvent la prééminence et la mission de mettre en œuvre l'intérêt public. On vise à rétablir une hiérarchisation des moyens et des fins. Les notions de « sociétal » et de «social » sont ainsi réservées à ce plan de l'action collective. La notion de responsabilité sociétale implique qu'existent des principes directeurs permettant de sélectionner des microdécisions parmi d'autres, afin de réaliser les objectifs collectivement déterminés, en matière de développement, de croissance, d'expansion économique. La responsabilité sociétale implique la mise en application d'une approche de participation coopérative, intégrée dans une formule de mixed-scanning sur le plan décisionnel $^{19}$. L'objectif est ambitieux, étant 
donné la rareté des animateurs et des gestionnaires ayant reçu une formation appropriée, voire la rareté des médias capables de rendre compte et de faciliter de tels exercices collectifs. Sans oublier la dominance au sein des collectivités de forces puissantes peu intéressées à abandonner leurs situations privilégiées. La Norme pourrait de fait être adoptée formellement, mais sans le cadre institutionnel, sans les habiletés, sans les rapports de force qui la rendraient effective.

\section{Les articles 1 et 2 de la Norme}

ISO 26000 ne vise pas à se « substituer » aux obligations légales nationales ou aux normes internationales de comportement. Elle vise à compléter ou à inspirer les autres instruments et initiatives de responsabilité sociétale.

Les deux premiers articles précisent le domaine d'application de la responsabilité sociétale et définissent les principaux termes et expressions qui seront utilisés dans la norme (comportement éthique, gouvernance de l'organisation, partie prenante, développement durable, transparence, etc.). Ils rappellent que cette norme a pour vocation de mettre en avant une compréhension commune du périmètre de la responsabilité sociétale de toute organisation, tout en réaffirmant que le respect de la loi est une dimension fondamentale de cette responsabilité. Ainsi, ISO 26000 ne vise pas à se «substituer» aux obligations légales nationales ou aux normes internationales de comportement. Elle vise à compléter ou à inspirer les autres instruments et initiatives de responsabilité sociétale.

\section{L'article 3 de la Norme}

Cet article décrit les facteurs, conditions et domaines de la responsabilité sociétale :

La principale caractéristique de la responsabilité sociétale se traduit par la volonté de l'organisation, d'une part, d'intégrer des considérations sociales et environnementales dans ses prises de décision, et d'autre part, d'être en mesure de répondre des impacts de ses décisions et activités sur la société et l'environnement. Ceci implique un comportement à la fois transparent et éthique qui contribue au développement durable, respecte les lois en vigueur et soit en cohérence avec les normes internationales de comportement. Cela suppose également que la responsabilité sociétale soit intégrée dans l'ensemble de l'organisation, qu'elle soit mise en œuvre dans le cadre des relations que celle-ci entretient et qu'elle prenne en compte les intérêts des parties prenantes.

La Norme situe l'organisation dans le contexte du développement durable, ce qui comprend la santé et le bien-être de la société. L'objectif de la responsabilité sociétale vise le développement; cela implique d'aborder les facteurs économiques, sociaux et environnementaux ainsi que leur interdépendance, dans les activités et les prises de décision de l'organisation.

\section{L'article 4 de la Norme}

Cet article présente et explique les «sept principes » directeurs qui fondent la responsabilité sociétale. On entend par «principes », les fondements, les valeurs-pivots du comportement, d'une bonne gestion, d'une prise de décision. Pour une organisation, on propose les principes suivant : 1) la redevabilité (ou reddition de compte) quant aux impacts qu'elle exerce sur la société et l'environnement; 2) la transparence des décisions qu'elle prend et des activités qu'elle mène lorsque celles-ci ont une incidence sur la société et l'environnement; 3) le comportement éthique qu'elle doit avoir en permanence; 4) la reconnaissance des intérêts des parties prenantes; 5) le respect du principe de légalité; 6) la prise en compte des normes internationales de comportement; 7) le respect des droits de l'Homme et la reconnaissance de leur universalité. À l'aide de ces sept principes, une organisation contribue de façon plus structurée au développement durable, y compris la santé et le bien-être de la société.

\section{L'article 5 de la Norme}

Cet article traite de deux pratiques fondamentales en matière de responsabilité sociétale. Il s'agit de l'identification par une organisation de ses responsabilités, de ses parties prenantes, 
et enfin du dialogue auquel celles-ci participent ou auquel idéalement elles devraient participer. L'organisation doit avoir conscience qu'elle existe en relation avec autrui, qu'elle est reliée en permanence à divers partenaires dans le jeu social. On sort donc de l'individualisme néolibéral pour proposer aux organisations une gouvernance plus collaborative, plus réflexive, qui reconnaît la valeur de la communauté.

\section{L'organisation doit avoir conscience qu'elle existe en relation avec autrui, qu'elle est reliée en permanence à divers partenaires dans le jeu social. On sort donc de l'individualisme néolibéral pour proposer aux organisations une gouvernance plus collaborative, plus réflexive, qui reconnaît la valeur de la communauté.}

\section{L'article 6 de la Norme}

L'article 6 explique le contenu substantif de la norme, en révèle le caractère original. Il invite les organisations à articuler leur démarche face à la responsabilité sociétale autour de sept questions centrales, soit la gouvernance de l'organisation (la redevabilité, la transparence, le comportement éthique, la reconnaissance des intérêts des parties prenantes, le respect du principe d'égalité, la prise en compte des normes internationales de comportement), les droits de l'Homme (naturels, inaliénables, universels, indivisibles, interdépendants), les relations et

\section{EFFETS DE LA NORME}

Sur le plan sociologique, l'effet sur les participants ne peut manquer de se produire. Les parties impliquées dans l'élaboration de la norme internationale ISO 26000 ont fait un travail remarquable de synthèse, équivalant à la construction d'un cadre conceptuel de sciences sociales, tout en réalisant une importante intervention à l'échelle planétaire. Comme le mode participatif d'élaboration de cette norme n'est pas habituel, y associant des représentants des travailleurs, des consommateurs, des ONG, etc., il y a là une méthodologie de construction coopérative du consensus qui parait répondre aux vœux du politicologue Robert Axelrod ${ }^{21}$. Pour Corinne Gendron, la «norme» va conditions de travail (la non-marchandisation du travail), l'environnement (principe de précaution, gestion du risque environnemental, principe de pollueur-payeur), la loyauté des pratiques des affaires (comportement éthique), les questions relatives aux consommateurs et l'engagement sociétal (appui et construction d'une relation avec les communautés) ${ }^{20}$. Chacune de ces questions se trouve redéployée selon un éventail de domaines d'action qui permettent à toutes les organisations d'identifier les principaux impacts qu'elles exercent sur la société.

Ces questions et domaines d'action doivent être considérés de manière holistique et en interdépendance; cela exclut de pouvoir focaliser la réflexion et son action uniquement par rapport à un domaine spécifique.

\section{L'article 7 de la Norme}

Enfin, l'article 7 précise les lignes directrices destinées à aider les organisations à intégrer concrètement la responsabilité sociétale dans leur manière d'opérer. Ces lignes directrices servent donc à appréhender et à intégrer la responsabilité sociétale dans l'organisation, à en améliorer la communication, à accroitre la crédibilité de l'organisation en cette matière, à revoir et à améliorer les actions et les pratiques à ce sujet et enfin à évaluer l'intérêt d'initiatives volontaires dont une liste non exhaustive se trouve présentée à l'annexe A de la Norme.

certainement alimenter les actions d'organisations désireuses de représenter les "intérêts » de mouvements sociaux, et présenter un véritable domaine d'amarrage de leur action ${ }^{22}$.

Sur le plan juridique, ISO 26000 ne constitue pas une norme applicable par les tribunaux nationaux (certains auteurs parlent de soft law ou droit mou), ni certifiable par une organisation de normalisation. Mais on peut déjà anticiper que des cours de justice pourront s'y référer comme cadre balisant les actions, comme un ensemble «prédroit » de valeurs communes, de coutumes à l'échelle mondiale. C'est révolutionnaire, car ce texte dont le contenu porte sur un ensemble de 
normes ou d'attentes (les droits collectifs) est traduit en 16 langues, ce qui va lui assurer une diffusion planétaire. De plus, il n'est pas exclu que les législatures nationales puissent être influencées par l'esprit et la lettre de la norme ISO 26000 lors de la rédaction de leurs textes de lois ${ }^{23}$.

Ainsi, des organismes nationaux de normalisation pourraient s'en inspirer comme ce fut le cas au Danemark avec la création de la norme nationale DS $26001^{24}$. Au Québec, l'OBNL Neuvaction ${ }^{25}$, le Bureau de normalisation du Québec (BNQ), la Chaire Desjardins en gestion du développement durable (Université de Sherbrooke), le ministère du Développement économique, de l'innovation et de l'exportation (MDEIE) et une vingtaine d'experts ont élaboré un référentiel d'application du développement durable en entreprise, qui vise à encourager et à faciliter l'application des principes de la Loi québécoise sur le développement durable dans tous les types d'organisations ${ }^{26}$. Le nouveau guide BNQ 21000, qui a été élaboré en cohérence avec ISO $26000^{27}$, permet aux entreprises et organisations d'établir le portrait de leurs pratiques et d'envisager comment elles se situent par rapport aux 21 enjeux du développement durable; ceux-ci se trouvent classés comme les 7 thèmes centraux

\section{CONCLUSION}

ISO 26000, un cadre volontaire ne peut remplacer le cadre juridique et institutionnel national ou international qui obligerait les entreprises à prendre en compte la société dans son ensemble, à répondre de ses actes à l'égard d'autrui, c'est-à-dire sur les hommes et la nature.

Il faut bien avoir conscience que les effets d'ISO 26000 sur la responsabilité sociétale des organisations et des entreprises (surtout multinationales) auront des limites. ISO 26000, un cadre volontaire ne peut remplacer le cadre juridique et institutionnel national ou international qui obligerait les entreprises à prendre en compte la société dans son ensemble, à répondre de ses actes à l'égard d'autrui, c'est-à-dire sur les hommes et la nature. Alain Supiot rappelle que pour être responsable sur le plan juridique, trois conditions sont indispensables. «La responsabilité implique
d'ISO 26000, en fonction des diverses parties prenantes $^{28}$. Il s'agit là d'une influence directe d'ISO 26000 sur notre réalité immédiate ${ }^{29}$.

Si au plan du management, ISO 26000 ne se présente pas comme une norme de gestion, on pourra s'en inspirer pour modifier la gouvernance des organisations, en particulier dans les calculs de risque, les procédures de gouvernance relatives aux nombreuses parties prenantes et la gestion de projet. L'élargissement de la matrice décisionnelle pourrait obliger à repenser la formation des gestionnaires, le cadre interdisciplinaire de formation en milieu universitaire, la formation continue des professionnels.

\section{Si au plan du management, ISO 26000 ne se présente pas comme une norme de gestion, on pourra s'en inspirer pour modifier la gouvernance des organisations, en particulier dans les calculs de risque, les procédures de gouvernance relatives aux nombreuses parties prenantes et la gestion de projet.}

une relation ternaire entre trois personnes: la personne du responsable, qui est le point causal d'imputation de l'acte; la personne du demandeur, dont les intérêts sont affectés par cet acte; et la personne du tiers (juge ou arbitre) devant qui le responsable doit répondre de son acte» ${ }^{30}$. Sur le plan global, la responsabilité implique de plus de répondre à un «appel », celui qui nous demande de construire un monde vivable et plus équitable ${ }^{31}$.

Force est de constater que, sur le plan mondial, il n'y a pas de citoyenneté, pas de personnes morales correspondant aux firmes ou réseaux de firmes, ni État ou Cour de justice habilitée à juger de leurs rapports. Dans l'état actuel des relations internationales, bien des éléments du cadre institutionnel de développement font gravement défaut, et leur installation rencontre des forces très puissantes vouées à l'affaiblissement de tels éléments. ISO 26000, malgré ses limites, représente une force active de changement pour la mise 
en place d'un ordre mondial dans lequel les éléments essentiels de la responsabilité trouveront une place qu'ils n'ont pas toujours occupée au plan national..

\section{ISO 26000, malgré ses limites, représente une force active de changement pour la mise en place d'un ordre mondial dans lequel les éléments essentiels de la responsabilité trouveront une place qu'ils n'ont pas toujours occupée au plan national.}

\section{BIBLIOGRAPHIE ET NOTES}

${ }^{1}$ Alain Supiot, L'esprit de Philadelphie. La justice sociale face au Marché total, Paris, Seuil, 2010; Alain Supiot, «Contribution à une analyse juridique de la crise économique de $2008 »$, Revue internationale $d u$ travail, vol. 149, 2010, no 2, p. 165-176; Eddy Lee, «La Déclaration de Philadelphie: rétrospective et prospective », Revue internationale du travail, Vol. 133, no 4, 1994, 513-531.

${ }^{2}$ Alain Supiot, «Du nouveau au self-service normatif : la responsabilité sociale des entreprises », dans Études offertes à Jean Pélissier. Analyse juridique et valeurs en droit social, Paris, Dalloz, 2004, p. 541-558, 543.

${ }^{3}$ Andrew Crane, Dirk Matten, Business Ethics. Managing Corporate Citizenship and Sustainability in the Age of Globalization, third edition, Oxford University Press, New York, 2010.

${ }^{4}$ Alain Supiot, «Du nouveau au self-service normatif : la responsabilité sociale des entreprises », dans Études offertes à Jean Pélissier. Analyse juridique et valeurs en droit social, Paris, Dalloz, 2004, p. 541558, 543. Il est important de rappeler qu'avec la globalisation des marchés, il n'y a aucune règle instituant les aspects sociaux et environnementaux de cette libéralisation (ex. les conventions de l'OIT). Pourtant, il y a des règles instituant la libéralisation internationale du commerce qui s'imposent aux ordres juridiques nationaux.

${ }^{5}$ Jacques Racine, «La responsabilité sociale de l'entreprise: un concept polymorphe », dans De la responsabilité sociale. Éthique et politique, Éric Gagnon et Francine Saillant, Montréal, Liber, p. 125142.

${ }^{6}$ Philippe Chalmin, Le monde a faim, éditions Bourin, 2009. On lira aussi à ce sujet : Joseph Stiglitz, Le triomphe de la cupidité, Paris, Fayard, 2010; Jean Ziegler, Destruction massive. Géopolitique de la faim, Paris, Seuil, 2011; Michel Freitag, L'impasse de la globalisation. Une histoire sociologique et philosophique du capitalisme, Montréal, Les Éditions Écosociété, 2008.
${ }^{7}$ Milton Friedman, «The Social Responsability of Business Is to Increase its Profits », The New York Times Magazine, 13 septembre 1970, p. 32-33, 122124; Milton Friedman, La liberté de choix, Paris : Pierre Belfond, 1980; Friedrich von Hayek, Droit, législation et liberté : une nouvelle formulation des principes libéraux de justice et d'économie politique, Coll. Libre échange, Paris, Presses universitaires de France, 1980-1983.

${ }^{8} \mathrm{Ce}$ qui est manifestement faux, sur le plan juridique, si l'on considère les lois nationales corporatives au Canada, la Loi canadienne sur les sociétés par actions, L.R.C. (1985), ch. C-44 en particulier, et les jugements des tribunaux sur cette question soit : Magasins à rayons Peoples inc. (Syndic de) c. Wise, [2004] 3 R.C.S. 461, 2004 CSC 68; BCE inc. c. Détenteurs de débentures de 1976, [2008] 3 R.C.S. 560, 2008 CSC 69.

${ }^{9}$ Corinne Gendron, «L'entreprise comme vecteur $d u$ progrès social : la fin ou le début d'une époque? », Cahier de la CRSDD, Coll. «Recherche", no 012009, Montréal : Chaire de responsabilité sociale et de développement durable, ESG UQAM, 22 p.

${ }^{10}$ Robert K. Merton, Éléments de théorie et de méthode sociologique, Paris, Gérard Monfort, 1965, (Chapitre IV et son appendice); sur la prophétie autoréalisatrice en matière économique, on consultera Bernard Maris, Anti-manuel d'économie, Paris, Éditions Bréal, 2003, p. 306 et suiv.

${ }^{11}$ Comme on l'a constaté dans les crises de 1929 et de 2008 ou récemment encore en Angleterre, en Espagne et en Grèce, la rumeur de faillite ou de défaut d'une banque peut avoir pour effet d'entraîner une ruée sur les guichets, précipitant ainsi le défaut de paiement. La prophétie de faillite, qui peut être une fausseté logique au départ, fait arriver ce qu'elle annonce en se propageant dans son public. La solution est d'établir une garantie de solvabilité, ou assurance-dépôt, de l'État concerné.

${ }^{12}$ On lira entre autres à ce sujet : Joseph E. Stiglitz, Un autre monde. Contre le fanatisme du marché, Paris, Fayard, 2006; Amartya Sen, L'idée de justice, Paris, Flammarion, 2010; Alain Supiot, L'esprit de Philadelphie. La justice sociale face au Marché total, Paris, Seuil, 2010.

${ }^{13} \mathrm{La}$ lingua franca fut une langue pratiquée par les nations commerçantes autour de la Méditerranée (Venise, Turquie, etc.) entre l'époque des croisades et la fin $\mathrm{du} \mathrm{XIX}^{\mathrm{e}}$ siècle. On définit actuellement cette expression comme «une langue auxiliaire de relation utilisée par des groupes ayant des langues maternelles différentes ». http://www.larousse.fr/dictionnaires/ francais/lingua_franca/47264.

${ }^{14}$ Karim Benyekhlef, Une possible histoire de la norme. Les normativités émergentes de la mondia-lisation, Montréal, Les Éditions Thémis, 2008, p. 776 
${ }^{15}$ Michel Capron, Françoise Quairel, Marie-France Turcotte, ISO 26 000, une norme " hors norme »? : vers une conception mondiale de la responsabilité sociétale, Paris, Economica, 2011.

${ }^{16}$ Corinne Gendron, «ISO 26000 : vers une définition socialement construite de la responsabilité sociale d'entreprise », Cahier de la Chaire-collection recherche, Montréal, ESG, UQAM, no 02-2009, 9 p.

${ }^{17}$ Dans le cadre «conceptuel» de l'utopie néolibérale, le travail, la nature, la monnaie ne sont que des marchandises parmi d'autres. Même le droit est considéré « comme un produit en compétition à l'échelle du monde, où s'opérerait la sélection naturelle des ordres juridiques les mieux adaptés à l'exigence de rendement financier ». Alain Supiot, L'esprit de Philadelphie. La justice sociale face au Marché total, Paris, Seuil, 2010, p. 64; On lira aussi à ce sujet, du même auteur, "Le droit du travail bradé sur le marché des normes », Droit social, 2005, p. 1087 et suiv.

${ }^{18}$ La Déclaration de Philadelphie a été incorporée en 1946, dans son intégralité, en tant qu'annexe, à la constitution de l'Organisation internationale du travail (OIT).

${ }^{19}$ Amitaï Etzioni, The Active Society, New York, The Free Press, 1968; Amitaï Etzioni, A Sociological Reader on Complex Organizations, New York, Holt, Rinehart and Winston Inc., 1969; Amitaï Etzioni, «Humble Decision Making », Harvard Business Review, Vol. 89, 1989, 122.

${ }^{20} \mathrm{La}$ norme mentionne que d'autres domaines d'action peuvent apparaître à l'avenir, car la responsabilité sociétale est une notion dynamique qui reflète l'évolution des préoccupations sociétales et environnementales.

${ }^{21}$ Robert Axelrod, Comment réussir dans un monde d'égoïstes? Théorie du comportement coopératif, Odile Jacob, Paris, 2006.

${ }^{22}$ Corinne Gendron, «Normaliser la responsabilité sociale : Le pari d'ISO $26000 »$, Les cahiers de la CRSDD, No 07-2010, 72 p.

${ }^{23}$ À la suite de son expérience lors de sa participation à l'élaboration de la norme ISO 26000, la République d'Indonésie a créé, en 2007, une loi s'en inspirant, la loi no 40 sur les sociétés à responsabilité limitée. Nugroho, S., Sintia, E., Widyana, N. Mise en ouvre d'ISO 26000 : avantages et attentes. Page consultée le 6 avril 2012: http://www.iso.org/iso/fr/iso_ catalogue/management_and_leadership_standards/so cial_responsibility/sr_news-and-articles/sr_contest/sr contest text8.htm.

${ }^{24}$ Michel Capron, «Légitimité et crédibilité des lignes directrices ISO $26000 »$, dans ISO 26000 , une norme "hors norme»? : vers une conception mondiale de la responsabilité sociétale, Paris, Economica, 2011, p. 37-54, 51.

${ }^{25}$ «Organisme à but non lucratif (OBNL), Neuvaction est l'une des deux entreprises agréées au Canada par la Global Reporting Initiative (GRI) et autorisée à donner la formation certifiée, en français et en anglais, en matière de reporting et de reddition de comptes du développement durable et de la responsabilité sociétale des entreprises et organismes selon la méthodologie GRI, méthodologie reconnue à l'échelle internationale ». http://www.bnq21000. qc.ca/projet-bnq-21000/le-projet-bnq-

21000/partenai-res-strategiques/

${ }^{26}$ Développement durable.com. (2011). Guide BNQ 21000 : développement durable pour les entreprises québécoises. [En ligne]. Adresse URL : http://www.xn--dveloppementdurable-bzb.com/ category/nouvelles/bnq-21000 (Page consultée le 26 juin 2012).

${ }^{27}$ Mais aussi en cohérence avec le SD 21000 de l'AFNOR en France, les lignes directrices pour la rédaction de rapport de développement durable de la Global Reporting Initiative (GRI) et les principes du Pacte mondial des Nations Unies.

${ }^{28}$ Karine Casault, «Forte adhésion au projet BNQ 21000 », Gä̈a Presse, $1^{\text {er }}$ juin 2012.

29 Un sondage du MDEIE 2009-2010 sur le développement durable (DD) à l'intention des entreprises québécoises - 3353 répondants - révèle que: «Actuellement, entre $10 \%$ et $20 \%$ des entreprises québécoises ont amorcé une démarche de développement durable. »

${ }^{30}$ Alain Supiot, «Du nouveau au self-service normatif : la responsabilité sociale des entreprises », dans Études offertes à Jean Pélissier. Analyse juridique et valeurs en droit social, Paris, Dalloz, 2004, p. 541-558, 549

${ }^{31}$ Guillaume De Stexhe, «Devoir, pouvoir? La responsabilité dans les limites de la simple humanité », Hugues Dumont, François Ost \& Sébastien van Drooghenbroeck Sébastien (éds.), La responsabilité face cachée des droits de l'homme, Bruxelles, Bruylant, 2005. 